March $2000 \quad$ NREL/CP-520-27747

\title{
Direct Measurement of
} Density-of-States Effective Mass and Scattering Parameter in Transparent conducting Oxides Using Second-Order Transport Phenomena

D.L. Young and T.J. Coutts

National Renewable Energy Laboratory

V.I. Kaydanov

Colorado School of Mines: Golden, Colorado

W.P. Mulligan

Sunpower Inc.: Sunnyvale, California

Presented at the American Vacuum Society $46^{\text {th }}$ International Symposium

Seattle, Washington

October 24-29, 1999

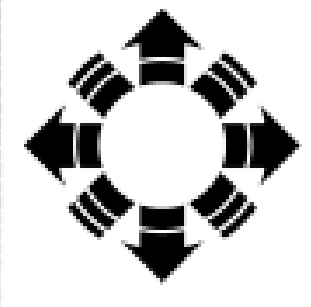

\section{NPEI}

National Renewable Energy Laboratory

1617 Cole Boulevard

Golden, Colorado 80401-3393

NREL is a U.S. Department of Energy Laboratory

Operated by Midwest Research Institute $\bullet$ Battelle $\bullet$ Bechtel

Contract No. DE-AC36-99-G010337 


\section{NOTICE}

The submitted manuscript has been offered by an employee of the Midwest Research Institute (MRI), a contractor of the US Government under Contract No. DE-AC36-99G010337. Accordingly, the US Government and MRI retain a nonexclusive royalty-free license to publish or reproduce the published form of this contribution, or allow others to do so, for US Government purposes.

This report was prepared as an account of work sponsored by an agency of the United States government. Neither the United States government nor any agency thereof, nor any of their employees, makes any warranty, express or implied, or assumes any legal liability or responsibility for the accuracy, completeness, or usefulness of any information, apparatus, product, or process disclosed, or represents that its use would not infringe privately owned rights. Reference herein to any specific commercial product, process, or service by trade name, trademark, manufacturer, or otherwise does not necessarily constitute or imply its endorsement, recommendation, or favoring by the United States government or any agency thereof. The views and opinions of authors expressed herein do not necessarily state or reflect those of the United States government or any agency thereof.

Available electronically at http://www.doe.gov/bridge

Available for a processing fee to U.S. Department of Energy and its contractors, in paper, from:

U.S. Department of Energy

Office of Scientific and Technical Information

P.O. Box 62

Oak Ridge, TN 37831-0062

phone: 865.576 .8401

fax: 865.576.5728

email: reports@adonis.osti.gov

Available for sale to the public, in paper, from:

U.S. Department of Commerce

National Technical Information Service

5285 Port Royal Road

Springfield, VA 22161

phone: 800.553 .6847

fax: 703.605.6900

email: orders@ntis.fedworld.gov

online ordering: http://www.ntis.gov/ordering.htm

Printed on paper containing at least $50 \%$ wastepaper, including $20 \%$ postconsumer waste 
Paper presented at the American Vacuum Society $46^{\text {th }}$ International Symposium in Seattle, WA.

October 24-29, 1999

Abstract Number: 1045

Program Number: TF-WeA10

Session Title: Transparent Conductive Oxides

\title{
Direct Measurement of Density-of-States Effective Mass and Scattering Parameter in Transparent Conducting Oxides Using Second-Order Transport Phenomena
}

D.L. Young, T.J. Coutts, National Renewable Energy Laboratory, Golden, CO

V.I. Kaydanov, Colorado School of Mines, Golden, CO.

W.P. Mulligan, Sunpower Inc. Sunnyvale, CA.

\begin{abstract}
:
The Boltzmann transport equation can be solved to give analytical solutions to the resistivity, Hall, Seebeck, and Nernst coefficients. These solutions may be solved simultaneously to give the density-of-states (DOS) effective mass $\left(m_{d}^{*}\right)$, the Fermi energy relative to either the conduction or valence band, and a scattering parameter that is related to a relaxation time and the Fermi energy. The Nernst coefficient is essential for determining the scattering parameter and, thereby, the effective scattering mechanism(s). We constructed equipment to measure these four transport coefficients simultaneously over a temperature range of 30-350 K for thin, semiconducting films deposited on insulating substrates. We measured these coefficients for $\mathrm{rf}$ magnetron-sputtered zinc oxide, both doped and undoped, with aluminum with carrier concentrations in the range of $1 \times 10^{19}-5 \times 10^{20} \mathrm{~cm}^{-3}$. The $\left(m_{d}^{*}\right)$ was not constant over this carrier concentration range: varying from 0.3 to $0.48 \mathrm{~m}_{\mathrm{e}}$, leading us to conclude that zinc oxide has a nonparabolic conduction band. Conductivity effective mass values for zinc oxide matched our $m_{d}^{*}$ values, revealing a single valley, nearly spherical, constant energy surface for zinc oxide. The measured scattering parameter changed from close to zero to 1.5 as the carrier concentrations increased. The scattering parameter, Seebeck, and mobility vs. temperature data support neutral impurity scattering in the undoped material and ionized impurity scattering in the Al-doped $\mathrm{ZnO}$. The transport theory also allows an extrapolation for an $m_{d}^{*}$ value at the bottom of the conduction band, which was found to be $0.27 \mathrm{~m}_{\mathrm{e}}$.
\end{abstract}




\section{Introduction}

Transparent conductive oxide (TCO), thin-film semiconductors are used in many large-volume and technically significant applications, ${ }^{1}$ yet they remain some of the least-understood materials, in part due to their relatively poor transport properties. TCO films represent a compromise between optical transmittance, bounded by absorbance at the optical band gap and reflectance at the plasma frequency, and electrical conductivity. As noted by Coutts et al., ${ }^{2}$ this compromise must pivot about maximizing the mobility. For highly degenerate, metal-like TCO films, mobility may be defined by the Drude theory, $\mu=q \tau / \mathrm{m}^{*}$, where $\mathrm{m}^{*}$ is the conductivity effective mass and $\tau$ is the time between carrier-scattering elastic collisions, known as the relaxation time. Obviously, either a long relaxation time or a small $\mathrm{m}^{*}$ will increase the mobility, but either quantity represents a formidable task to measure; the former is very difficult. Cyclotron and de Haas van Alphen resonance techniques, the traditional methods for probing the Fermi surface via the effective mass, are only applicable for high-mobility materials because of the constraint that the product $\mu \mathbf{B}>>1$ so that electrons complete at least one orbit before they are scattered. With typical magnetic fields in the laboratory being on the order of one Tesla, the mobility must be in the tens of thousands $\mathrm{cm}^{2} / \mathrm{V}$-s. TCOs, with their relatively small mobilities $\left(\mu<300 \mathrm{~cm}^{2} / \mathrm{V}\right.$-s $)$ and short relaxation times $\left(\sim 10^{-15}\right.$ s), do not meet the $\mu \mathbf{B}>>1$ requirement and are thus unsuitable for these measurement techniques. Effective mass values for polycrystalline, thin-film TCOs are usually inferred from optical modeling (plasma frequency, or transmission, reflection data) or assumed to be equal to single-crystal bulk values.

In addition to the need for a direct measure of $\mathrm{m}^{*}$ in TCOs and other polycrystalline thin films, understanding the mechanism for scattering of carriers in films is of great importance for improving transport properties. To better understand the band structure and the dominant scattering mechanisms, a novel measurement technique, based on transport phenomena, has been applied to TCO thin-films. Transport-phenomena coefficient measurements — resistivity, Hall, Seebeck, and Nernst (sometimes referred to as Nernst-Ettingshausen) — on thinfilm TCO samples were used with solutions to the Boltzmann transport equation to make direct measurements of the DOS effective mass, $m_{d}^{*}$, and an energy-dependent scattering parameter, s. We have constructed equipment to enable these measurements for films grown on electrically insulating substrates. We have applied this technique to TCO thin-film samples and have made, to our knowledge, the first direct measurements of $m_{d}^{*}$ and s on films of cadmium 
stannate, zinc oxide, tin oxide, and cadmium oxide. In this paper, we will discuss transport theory, our transport coefficient equipment, and data obtained from our zinc oxide study.

\section{Theory}

Transport, or motion of charge carriers in a material, is described by Boltzmann's transport equation, which relates changes in the carrier distribution (occupied energy states) to forces that drive the carriers to a nonequilibrium state. The carriers (electrons for n-type materials) in a degenerate material in equilibrium are described by the Fermi-Dirac distribution function, $f_{o}(\vec{k}, \vec{r})$. When external driving forces from temperature gradients or electric and magnetic fields are imposed on a material, the carriers are driven to higher energy (excited) states. The redistribution of occupied energy levels from the equilibrium configuration may be described by a non-equilibrium distribution function, $f(\vec{k}, \vec{r})$. Carriers in excited states will return to their equilibrium distribution after collision, scattering events between other charge carriers or imperfections in the material. ${ }^{3}$ Transport by electric and thermal current densities in a material are described by

$$
\vec{J}=\frac{q}{4 \pi^{3}} \int \vec{v} f(\vec{k}, \vec{r}) d k \quad \text { Amps } / \mathrm{m}^{2} \quad \vec{Q}=\frac{1}{4 \pi^{3}} \int\left(E-E_{F}\right) \vec{v} f(\vec{k}, \vec{r},) d k \quad \text { Watts } / \mathrm{m}^{2},
$$

where $\vec{v}$ is the carrier velocity. To solve these integrals, $f(\vec{k}, \vec{r})$ must be determined by solving the steady-state Boltzmann transport equation, 4

$$
\left(\frac{\partial f}{\partial t}\right) \approx \frac{q}{\hbar}(\vec{E}+\vec{v} \times \vec{B}) \cdot \vec{\nabla}_{k} f(\vec{k}, \vec{r})+\vec{v} \cdot \vec{\nabla}_{r} f(\vec{k}, \vec{r}),
$$

which relates the rate-of-change of the non-equilibrium function to driving forces acting on the non-equilibrium energy distribution of carriers. Obviously, this is a very difficult equation to solve because it requires knowledge of occupation and transition probabilities between excited and equilibrium states. Traditionally, a relaxation time approximation has been made to allow analytical solutions. This approximation assumes a mean time between collisions, when carriers are forced from an excited to an equilibrium distribution or vice versa - known as the relaxation time, $\tau$. The change in the distribution function can be approximated by

$$
\left(\frac{\partial f}{\partial t}\right) \approx\left(\frac{f_{0}-f}{\tau}\right) \quad 1 / \mathrm{sec} .
$$

The relaxation time between collisions is not a function of direction of the carrier motion, but is a function of energy and possibly temperature. ${ }^{3}$ The frequency of elastic collisions, $\tau^{-1}(E)$, depends on the density-of-states function, 
$\mathrm{g}(\mathrm{E})$, and the square of the matrix element of the scattering transition from one energy state to a final state of the same energy, w(E). For parabolic energy bands, $g(E) \propto E^{1 / 2}$, while w(E) is a power function of energy $w(E) \propto E^{-s} \propto\left(k^{2}\right)^{-s}$. Thus, $\tau^{-1}(E)=w(E) g(E)=E^{-s} E^{1 / 2}=E^{1 / 2-s}$, which is often written $\tau \propto E^{s-1 / 2} \propto E^{s^{\prime}}$ with s' $=\mathrm{s}-1 / 2$. For the parabolic band model, $\mathrm{s}^{\prime}$ has been identified for several scattering mechanisms that are:

$$
\begin{aligned}
& \begin{array}{l}
\text { Acoustic Phonon scattering } \longrightarrow s^{\prime}=-1 / 2, \quad s=0 \\
\text { Neutral Impurity scattering } \longrightarrow s^{\prime}=0, s=1 / 2
\end{array} \\
& \text { Ionized Impurity scattering } \longrightarrow s^{\prime}=3 / 2 \quad s=2
\end{aligned}
$$

In the non-parabolic energy-band case, the energy of the carriers can be described by the function

$$
\frac{\hbar^{2} k^{2}}{2 m_{d 0}^{*}}=\gamma(E)=E+\frac{E^{2}}{E_{1}}+\frac{E^{3}}{E_{2}}+\ldots
$$

where $\mathrm{E}_{1}$ and $\mathrm{E}_{2}$ are constants, which preserves the same transition matrix element dependence on $k^{2}$ as in the parabolic case. The non-parabolic density-of-states function, $g_{N . P .}(E) \propto \gamma^{1 / 2}\left(\frac{d \gamma}{d E}\right)$, leads to

$$
\tau_{N . P .}^{-1}=w(E)_{N . P .} g(E)_{N . P .} \propto \gamma^{-s} \gamma^{1 / 2}\left(\frac{d \gamma}{d E}\right),
$$

and finally,

$$
\tau \propto \gamma^{s-1 / 2}\left(\frac{d \gamma}{d E}\right)^{-1} \propto \gamma^{s^{\prime}}\left(\frac{d \gamma}{d E}\right)^{-1}
$$

Clearly, the s' values for different scattering mechanisms in the non-parabolic case are the same as in the parabolic case.

Many texts ${ }^{3-6}$ outline the solution of Boltzmann's equation for $f(\vec{k}, \vec{r})$ using the relaxation time approximation and further solve equations [1] for electric and thermal current densities. In general, equations [1] have the following form, ${ }^{3}$

$$
J_{\alpha}=\frac{q}{4 \pi^{3}} \int v_{\alpha}^{2} \tau G_{\alpha}(E) \frac{\partial f_{0}}{\partial E} d k \text { and } Q_{\alpha}=\frac{1}{4 \pi^{3}} \int\left(E-E_{F}\right) v_{\alpha}^{2} \tau G_{\alpha}(E) \frac{\partial f_{0}}{\partial E} d k
$$

where $G_{\alpha}(E)$ is a function of temperature gradients and electric and magnetic forces, and $\alpha$ represents different crystallographic directions. Equations [4] may be related to the phenomenological equations for carrier transport, 


$$
J_{\alpha}=\sigma_{\alpha \beta} E_{\beta}-\theta_{\alpha \beta} \frac{d T}{d x_{\beta}} \quad \text { and } \quad Q_{\alpha}=\chi_{\alpha \beta} E_{\beta}-\xi_{\alpha \beta} \frac{d T}{d x_{\beta}}
$$

to identify specific transport coefficients. In equations [5], $E_{\beta}$ is an electric field, $d T / d x_{\beta}$ is a temperature gradient across a sample, and $\sigma_{\alpha \beta}, \theta_{\alpha \beta}, \chi_{\alpha \beta}, \xi_{\alpha \beta}$ are transport tensors that depend on the orientation and strength of a magnetic field. ${ }^{7}$ To match experimental setups, temperature gradients and electric fields are placed in the $\mathrm{x}, \mathrm{y}$ plane, whereas magnetic fields are parallel to the z-axis. This arrangement leads to analytical solutions for conductivity, Hall, Seebeck, and Nernst coefficients. Integral equations for n-type material coefficients are

$$
\begin{aligned}
& \sigma=\frac{q^{2} n}{m^{*}}\langle\tau\rangle \quad \text { conductivity, } \quad R_{H}=\frac{-1}{n q} \frac{\left\langle\tau^{2}\right\rangle}{\langle\tau\rangle^{2}} \quad \text { Hall, } \\
& \left.\alpha=\frac{-1}{q T}\left[\frac{\langle\tau E\rangle}{\langle\tau\rangle}-E_{F}\right] \quad \text { Seebeck, } \quad Q=\frac{-\mu_{n}}{q T}\left[\frac{\left\langle\tau^{2} E\right\rangle}{\langle\tau\rangle^{2}}-\frac{\left\langle\tau^{2}\right\rangle\langle\tau E\rangle}{\langle\tau\rangle^{3}}\right] \text { Nernst, } \quad\right\}
\end{aligned}
$$

where $\left\langle\tau^{m} E^{n}\right\rangle=\frac{\int_{0}^{\infty}\left(\tau^{m} E^{n}\right) E g(E) \frac{\partial f_{0}}{\partial E} d E}{\int_{0}^{\infty} E g(E) \frac{\partial f_{0}}{\partial E} d E}$ and $g(E)$ is a density-of-states function.

Kolodziejczak et al. ${ }^{7}$ first solved equations [6] for materials with ellipsoidal energy surfaces. Later, $\operatorname{Kaydanov}^{8}$ also $^{-1}$ solved the equations using the energy function described by equation [2]. When $\gamma(E)=E$, the (in general) nonparabolic energy bands become parabolic. $\gamma(E)$, then, describes the state of non-parabolicity for the energy bands in a material. Analytic expressions for equations [6] are solved using the method of Bethe-Sommerfeld ${ }^{9}$ to approximate the Fermi integrals. Keeping only lowest-order, non-vanishing terms, the conductivity and Hall coefficients require only first-order terms in the Sommerfeld expansion, whereas the Seebeck and Nernst coefficients require retention of second-order terms to first-order in temperature. The results for the Seebeck and Nernst coefficients for low magnetic fields $\mu \mathbf{B}<<1$ and isotropic materials of high degeneracy are

$$
\begin{aligned}
& \alpha_{0}=\frac{2}{3} \alpha_{\infty}\{s+1-\lambda\} \text { Seebeck coefficient } \\
& Q_{0}=\mu \frac{2}{3} \alpha_{\infty}\left\{s-\frac{1}{2}-\lambda\right\} \text { Nernst coefficient, }
\end{aligned}
$$


where $\lambda=2 \gamma\left(\frac{d \gamma}{d \varepsilon}\right)^{-2} \frac{d^{2} \gamma}{d \varepsilon^{2}}, \mathrm{~s}$ is the scattering parameter, and $\mu$ is the mobility. The term $\alpha_{\infty}=\frac{k_{0}^{2} T}{q \hbar^{2}}\left(\frac{\pi}{3 n}\right)^{2 / 3} m_{d}^{*}$ is the Seebeck coefficient under high magnetic field $(\mu \mathbf{B}>>1)$ and $m_{d}^{*}=m_{d 0}^{*} \frac{d \gamma}{d \varepsilon}$ is the energy-dependent DOS effective mass, with $m_{d 0}^{*}$ representing the DOS effective mass at the conduction-band minimum. Notice that the second term within the braces for equations [7] and [8], $\lambda$, depends on the energy bands of the material and vanishes for parabolic-band materials when $\gamma(E)$ is constant. Kolodziejczak et al. ${ }^{10}$ noticed that the high field Seebeck effect could be used to probe the energy band of a material without knowledge of the scattering mechanism. Dubrovskaya et al. ${ }^{11}$ investigated single-crystal $\mathrm{PbTe}$ in this manner. Unfortunately, this technique is not applicable for TCOs, again, because of their low mobilities and the $\mu \mathbf{B}>>1$ requirement for high field measurements.

Zhitinskaya et al. ${ }^{12}$ were the first to recognize that equations [7] and [8] could be combined to give equations for the effective density-of-states mass at the Fermi level,

and s, the scattering parameter,

$$
\begin{aligned}
& m_{d}^{*}=\left(\frac{3 n}{\pi}\right)^{2 / 3} \frac{q \hbar^{2}}{k_{B}^{2} T}\left(\alpha-\frac{Q}{\mu}\right), \\
& s=\frac{3}{2} \frac{(Q / \mu)}{(\alpha-Q / \mu)}+\frac{1}{2}+\lambda .
\end{aligned}
$$

Substituting into [9] $\mu=\left|R_{H}\right| \sigma$ and $n \approx 1 / R_{H} q$, where $R_{H}$ is the Hall coefficient, we obtain

$$
m_{d}^{*}=\left(\frac{3}{\left|R_{H}\right| q \pi}\right)^{2 / 3} \frac{q \hbar^{2}}{k_{B}^{2} T}\left(\alpha-\frac{Q}{\left|R_{H}\right| \sigma}\right)
$$

which illustrates that the DOS effective mass may be experimentally determined by measuring the conductivity, Hall, Seebeck, and Nernst coefficients of a sample. This technique has been coined the method of four coefficients 8 and was originally applied to p-type PbTe single crystals. ${ }^{13}$ Our group has applied the method of four coefficients to thin-film TCO samples, using a specially designed instrument to measure the four coefficients on the same sample. 


\section{Experimental Procedure}

Our four-coefficient instrument for thin films has already been discussed in detail, ${ }^{14}$ but a brief review of its operation is in order. Measurement of four transport coefficients on thin-films has been attempted by other groups ${ }^{15}$ and emphasizes the need for a clever pattern in the film to accomplish all of the measurements on the same sample. For our machine, thin-film TCO samples are deposited on electrically insulating substrates and photolithographically etched to the pattern shown in Figure 1. The high aspect ratio of $\mathrm{W}<<\mathrm{L}$ conforms to the specimen shape dictated by the ASTM designation F76-86 16 for van der Pauw resistivity and Hall measurements, as well as ensuring a large Nernst voltage between contacts 1 and 3 and a large temperature gradient between contacts 2 and 4 . As depicted in Figure 1, the film is placed film-side down across two copper heater blocks, with contacts 2 and 4 making ohmic contact with the heater blocks by silver paint or an indium dot. Contacts 1 and 3 are indium-soldered to fine copper wire. The heater blocks are electrically isolated from each other, and each has a copper wire attached to it to make electrical contact to the film. The heater blocks have a differential thermocouple mounted between them to measure the temperature gradient across the sample. Heater block 2 has an additional embedded thermocouple for absolute temperature measurement. The entire sample holder of Figure 1 is cooled by a closed-cycle helium cryostat for temperature-dependent measurements from $30-350 \mathrm{~K}$.

To measure the four coefficients, a specific data collection sequence is followed, as depicted in Figure 2. Resistivity is measured by the van der Pauw method, i.e., biasing contacts 3 and 4 while measuring the voltage across contacts 1 and 2, as per ASTM designation F 76-86. The Hall coefficient is measured by biasing contacts 1 and 3 while a z-directed magnetic field (Fig. 1) is applied and measuring the voltage across contacts 2 and 4. Again, the Hall coefficient measurement is specified by ASTM designation F 76-86. The thermoelectric, or Seebeck, coefficient is measured by establishing a temperature gradient between contacts 2 and 4 and measuring the voltage developed between the same two contacts. This measurement requires a calibration with a material of known absolute Seebeck coefficient because the TCO thin-film and the sample holder itself form a thermoelectric couple. $\mathrm{Pb}$ and $\mathrm{Pt}$ were used to calibrate our instrument, which allows a direct measure of the absolute Seebeck coefficient of the thin-film sample. Finally, the Nernst coefficient is measured by maintaining the temperature gradient between contacts 2 and 4 while applying the z-directed magnetic field. A Nernst voltage is established between contacts 1 and 3. 


\section{Transport Phenomena}

A brief review of the four transport phenomena of interest will be helpful to our understanding of the results presented in the next section. Figure 3a depicts cases for the Hall, Seebeck, and Nernst coefficients for n-type, isotropic, parabolic energy-band materials, exhibiting ionized impurity scattering. The Hall effect is a measure of the deflection of the drift carriers in a magnetic field. This deflection is measured by the Hall angle, $\phi$. The magnitude of $\phi$ depends on the scattering mechanism through the relaxation time, $\tau,([3])$ whereas its orientation is sensitive to carrier type. The Seebeck effect is an electric field established under steady-state conditions between diffusion current from a temperature gradient and the counteracting drift current. The direction of the field is carrier-type dependent. The Nernst effect is analogous to the Hall effect except that the carriers are driven by a temperature gradient rather than by an applied bias. Carriers originating from the hot end of the sample will, on average, have more energy and have a higher velocity with respect to carriers from the cold end. The magnetic field will deflect the slower-moving carriers into a shorter-radius orbit than the faster-moving carriers and will establish a net charge, and thus, a Nernst electric field as shown. ${ }^{5}$ The Nernst field is not carrier-type sensitive, but is, as will be shown next, highly dependent on the scattering mechanism.

Figure $3 \mathrm{~b}$ depicts the path of an electron in crossed electric and magnetic fields. The net path or drift velocity is shown as a result of scattering events between the electron and the lattice. The angle $\phi$ is shown with respect to the electric field. The time between collisions, $\tau$, is proportional to the arc length of the orbit. If the relaxation time, $\tau$, is short for a material, the Hall angle, $\phi$, will be smaller than for a material with a longer relaxation time. Consider Figure 3c showing the Nernst effect for three different scattering mechanisms for n-type, isotropic, parabolic energy-band materials. The left figure shows the Nernst effect for ionized impurity scattering. Note that because $\mathrm{s}^{\prime}>0$, the relaxation time, and hence the Hall angle, must be larger for the more energetic (hot) electrons.

This condition gives a Nernst electric field directed to the right. Following Gerlach's sign convention, ${ }^{5}$ this configuration would give a positive Nernst coefficient. The center cartoon of Figure $3 \mathrm{c}$ depicts scattering by acoustic phonons. This time $\mathrm{s}^{\prime}<0$, making the relaxation time and the Hall angle larger for the less energetic (cold) electrons, establishing a Nernst electric field directed to the left and a negative Nernst coefficient. The right-most cartoon of Figure $3 c$ shows that if scattering is by neutral impurities $\left(s^{\prime}=0\right)$, then both Hall angles are equal and no Nernst 
electric field is established. The Nernst effect gives a clear signal for the type of scattering occurring in a parabolic band material by changing the sign of the Nernst voltage.

It is interesting to note that if the $\gamma(\varepsilon)$ function in [7] and [8] is not constant (non-parabolic energy bands), then the last term in [7] and [8] could become large enough to change the sign of the Seebeck and Nernst coefficients. This condition was first observed in n-type InSb, ${ }^{10}$ and it has appeared in our work with CdO,[Coutts, 2000 \#96] where the effective mass changes by a factor of three over an order of magnitude increase in carrier concentration. Because the degree of non-parabolicity of the energy bands can affect the sign of the Nernst effect, one must be careful in drawing conclusions about the scattering mechanism based solely on the sign of the Nernst coefficient, until the band shape has been mapped with a variety of carrier concentration samples. The last term in equations [7] and [8] may be shown to equal

$$
\left|2 \gamma\left(\frac{d \gamma}{d \varepsilon}\right)^{-2} \frac{d^{2} \gamma}{d \varepsilon^{2}}\right|_{E_{F}}=3 \frac{n}{m_{d}^{*}} \frac{d m_{d}^{*}}{d n}=3 \frac{d \operatorname{Ln}\left[m_{d}^{*}\right]}{d \operatorname{Ln}[n]}=\lambda, \quad \text { [12] }
$$

which allows direct measurement of the degree of non-parabolicity of a material once $m_{d}^{*}$ is known (via equation [11]) for a number of samples of different carrier concentrations.

\section{Results}

Our group has applied the method of four coefficients to several TCO thin-films grown under a variety of conditions. In this paper, we will present our preliminary findings on rf magnetron sputtered, undoped zinc oxide, and zinc oxide doped with aluminum thin-films. Prepared samples were photolithographically patterned to the shape shown in Figure 1 with film thickness measured by profilometry. The four relevant transport phenomena coefficients (conductivity, Hall, Seebeck, and Nernst) were measured by the method and by the instrument described above, and they were then used in equation [11] to calculate the DOS effective mass, $m_{d}^{*}$. Figure 4 shows the $m_{d}^{*}$ values plotted against the carrier density, n. A best-fit line was drawn through the points, which was then used to calculate $\lambda$ in

equations [10] and [12]. We first note that $m_{d}^{*}$ is not constant with carrier density and varies from about 0.3 to 0.45 as the carrier density changes from $2 \times 10^{19}-5 \times 10^{20} \mathrm{~cm}^{-3}$. This trend shows that the conduction energy band for $\mathrm{ZnO}$ is non-parabolic in this carrier density range and will make the energy-band term, $\lambda$, non-zero in equations 
[7],[8], and [10]. In addition to our $m_{d}^{*}$ values for $\mathrm{ZnO}$ plotted in Figure 4, conductivity effective-mass values are plotted, as well. These values were taken from the literature ${ }^{17,18}$ on plasma frequency-calculated conductivity effective masses. Note that the conductivity and DOS effective mass are in good agreement with each other, but that the error bars on the DOS effective mass are much smaller. Recalling that the relationship between the two effectivemass values is given by $m_{d}^{*}=N^{2 / 3}\left(m_{1}^{*} m_{2}^{*} m_{3}^{*}\right)^{1 / 3}$, we conclude that $\mathrm{N}$, the number of ellipsoids of revolution, must be one and that the conductivity effective-mass values $\left(m_{1}^{*} m_{2}^{*} m_{3}^{*}\right)$ for different crystallographic directions must be approximately equal. This being the case, we may conclude that $\mathrm{ZnO}$ has a single-valley minimum in the conduction energy band and that the constant energy surface must be spherical to within experimental uncertainty. The singlevalley minimum of $\mathrm{ZnO}$ is predicted by theory, ${ }^{19}$ but the uncertainty in the plasma frequency data makes it difficult to be certain of the spherical nature of the energy surface. We note that $\mathrm{ZnO}$ is hexagonal and that our films have a (200) orientation. Anisotrophy in the effective mass is not found in the literature, nor is it observed in the mobility above $100 \mathrm{~K} .20$

With $m_{d}^{*}$ known as a function of $\mathrm{n}$, we solve for $\lambda$ to determine the degree to which the band shape affects the scattering parameter, s, in equation [10]. Figure 5a shows the calculated scattering parameter values from equation [10] for our $\mathrm{ZnO}$ samples, along with the predicted trends in the scattering parameter for three different scattering mechanisms. For the $\mathrm{ZnO}: \mathrm{Al}$ samples, the measured scattering parameter matches well with the trend expected for ionized impurities. This result is expected for these films, where aluminum is added to dope the films ntype by contributing an electron to the conduction band, leaving behind an ionized aluminum atom. For the undoped material, the scattering parameter is found to lie most closely with the neutral impurity trend. To further support our claim that neutral and ionized impurity scattering are the main scattering mechanisms in $\mathrm{ZnO}$ and $\mathrm{ZnO}: \mathrm{Al}$, respectively, Figure 5b shows our Seebeck measurements plotted against expected Seebeck values for three scattering mechanisms. Again, ionized and neutral impurity scattering are the apparent mechanisms. Mobility vs. temperature data for both a $\mathrm{ZnO}$ and $\mathrm{ZnO}: \mathrm{Al}$ film are shown in Figure 5c. The undoped $\mathrm{ZnO}$ film shows a clear positive dependence of mobility on temperature, indicative of neutral impurities. As the temperature decreases, the number of neutral impurity centers will increase, decreasing the relaxation time between collisions, and thus decreasing the mobility. ${ }^{6}$ For the doped material, the mobility is seen to be almost constant with temperature, 
characteristic of ionized impurity scattering. ${ }^{4}$ A comparison of optical and Hall mobilities is needed to rule out grain-boundary scattering in the undoped films.

In addition to providing the DOS effective mass and scattering parameter values, the method of four coefficients may also be used to extrapolate $m_{d 0}^{*}$, the effective mass at the conduction-band minimum, and to measure the Fermi energy level relative to the conduction band. Equation [2] describes the energy function used for solving the transport equations, allowing a first-order non-parabolicity approximation to be defined by keeping only the first two terms in the energy expansion. Using the relations

$$
\begin{aligned}
& m_{d}^{*}=m_{d 0}^{*} \frac{d \gamma(E)}{d E} \text { and } n=\frac{8 \pi}{3} \frac{\left(2 m_{d 0}^{*}\right)^{3 / 2}}{h^{3}} \gamma^{3 / 2}, \text { one can solve for } \\
& \left(m_{d}^{*}\right)^{2}=\left(m_{d 0}^{*}\right)^{2}+4 \frac{m_{d 0}^{*}}{E_{1}}\left\lfloor\left.\left(\frac{3}{8 \pi}\right)^{2 / 3} \frac{h^{2}}{2}\right|^{2 / 3} .\right.
\end{aligned}
$$

Equation [13] shows that the y-intercept of a plot of $\left(m_{d}^{*}\right)^{2}$ vs. $n^{2 / 3}$ will reveal $m_{d 0}^{*}$, and the slope will define the constant, $\mathrm{E}_{1}$. Figure 6 shows a plot of $\left(m_{d}^{*}\right)^{2}$ vs. $\mathrm{n}^{2 / 3}$ with a $m_{d 0}^{*}$ value of $0.27 \mathrm{~m}_{\mathrm{e}}$. Note that the plot is linear, indicating that our first-order, non-parabolicity assumption is valid. In addition, the Fermi energy level, with respect to the bottom of the conduction band, can be defined by $E_{F e r m i}=\left(\frac{3}{8 \pi}\right)^{2 / 3} \frac{h^{2}}{2 m_{d 0}^{*}} n^{2 / 3} \frac{1}{(1+\beta)}$, where $\beta=\left(\frac{m_{d}^{*}(E)}{2 m_{d 0}^{*}}-\frac{1}{2}\right)$. Figure 4 , showing the calculated Fermi energy level above the conduction band vs. the carrier concentration, reveals a strong degeneracy in the doped samples $\left(\mathrm{E}_{\mathrm{Fermi}}-\mathrm{E}_{\text {conduction }}>0.5 \mathrm{eV}\right)$, but only a slight degeneracy in the undoped samples.

\section{Conclusions}

The method of four coefficients offers a powerful experimental technique for the TCO thin-film community for both mapping the energy bands via the effective mass and determining scattering parameters. Its benefit to our understanding of TCO films has been very encouraging. We plan to continue our study of TCOs, as well as other semiconducting thin-films, with this technique in hopes of furthering our understanding of the fundamental mechanisms in these materials. In particular, we hope to identify the scattering mechanisms responsible for limiting 
the all-important mobility. It is known that mobility limits both the electrical and optical properties of Drude

materials.

\section{Acknowledgements:}

The authors wish to thank Dr. Phillip A. Parilla, Dr. Timothy A. Gessert, and Mr. James Keane of the National Renewable Energy Laboratory and Mr. W. Grover Coors of the Colorado School of Mines for their contributions to this work.

\section{Bibliography}

${ }^{1}$ H. L. Hartnagel, A. L. Dawar, A. K. Jain, and C. Jagadish, Semiconducting Transparent Thin Films (Institute of Physics Publishing, London, 1995).

${ }^{2}$ T. J. Coutts, X. Wu, W. P. Mulligan, and J. M. Webb, Journal of Electronic Materials 25 (6), 935-943 (1996).

${ }^{3}$ W. F. Leonard, and J. T. L. Martin, Electronic Structure and Transport Properties of Crystals, First ed. (Robert E. Krieger Publishing Company, Malabar, FL, 1987).

${ }^{4}$ S. S. Li, Semiconductor Physical Electronics (Plenum, New York, 1993).

${ }^{5}$ E. Putley, The Hall Effect and Related Phenomena (Butterworths, London, 1960).

6B. M. Askerov, Electron Transport Phenomena in Semiconductors (World Scientific, Singapore, 1994).

${ }^{7}$ J. Kolodziejczak, and S. Zukotynski, Phys. Stat. Sol. 5 (145), 145-158 (1964).

${ }^{8}$ V. Kaydanov, Unpublished Lecture Notes, Colorado School of Mines, (1997).

${ }^{9}$ N. W. Ashcroft, and N. D. Mermin, Solid State Physics (Harcourt Brace College Publishers, Fort Worth, Texas, 1976).

10J. Kolodziejczak, and L. Sosnowski, Acta Phys. Polon. 21, 399 (1962).

${ }^{11}$ I. N. Dubrovskaya, and Y. I. Ravich, Soviet Physics-Solid State 8 (8), 1160-1164 (1966).

${ }^{12}$ M. K. Zhitinskaya, V. I. Kaidanov, and I. A. Chernik, Sov. Phys. - Sol. Stat. 8 (1), 295-297 (1966).

${ }^{13}$ I. A. Chernik, V. I. Kaidanov, N. V. Kolomoets, and M. I. Vinogradova, Sov. Phys. - Semi. 2 (6), 645-651 (1968).

${ }^{14}$ D. L. Young, T. J. Coutts, and V. I. Kaydanov, Review of Scientific Instruments 71 (2), 462-466 (2000).

${ }^{15}$ W. Jiang, S. N. Mao, X. X. Xi, X. Jiang, J. L. Peng, T. Venkatesan, C. J. Lobb, and R. L. Greene, Phys. Rev. Lett. 73 (9), 1291-1294 (1994).

16"'Standard Test Methods for Measuring Resistivity and Hall Coefficient and Determining Hall Mobility in SingleCrystal Semiconductors, F-76-86," in Annual Book of ASTM Standards (American Society of Testing and Materials, West Conshohocken, 1996).

${ }^{17}$ S. Brehme, F. Fenske, W. Fuhs, E. Nebauer, M. Poschenrieder, B. Selle, and I. Sieber, Thin Solid Films 342, 167173 (1999).

${ }^{18}$ K. H. Hellwege, "Landolt-Börnstein Numerical Data and Functional Relationships in Science and Technology: Semiconductors," in Numerical Data and Functional Relationships in Science and Technology, edited by O. Madelung, M. Schulz, and H. Weiss (Springer-Verlag Berlin-Heidelberg, Berlin, 1982), Vol. 17.

${ }^{19}$ S. Bloom, and I. Ortenburger, Phys. Stat. Sol. (b) 58 (561), 561-566 (1973).

${ }^{20} \mathrm{P}$. Wagner, and R. Helbig, J. Phys. Chem. Solids 35, 327-335 (1972). 


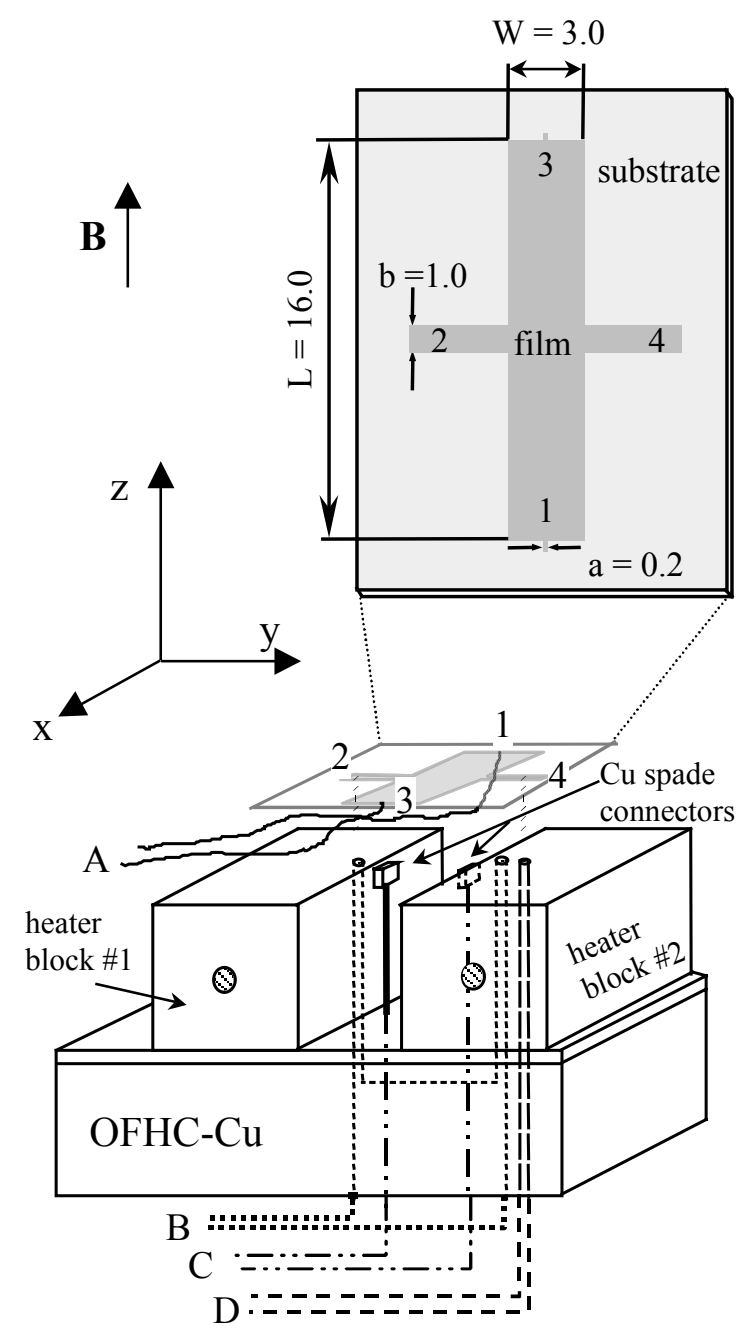

Fig. 1. Photolithographically etched thin-film sample on electrically insulating substrate placed film-side down across copper heater blocks. Lengths are in $\mathrm{mm}$. Wires A and C provide electrical contact to the sample, and differential thermocouple, $\mathrm{B}$, and thermocouple, $\mathrm{D}$, provide temperature information. 


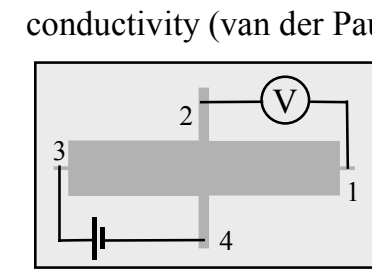

Seebeck effect

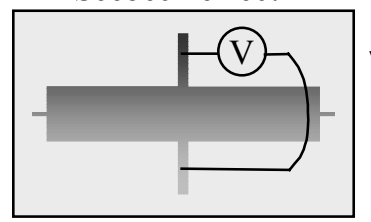

Hall effect

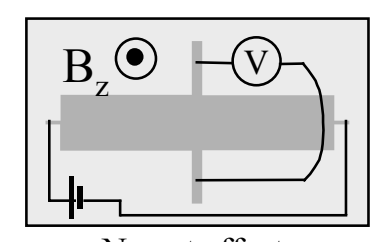

Nernst effect

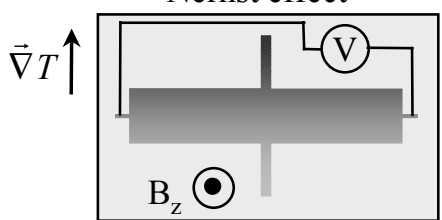

Fig. 2. Measurement configurations for collecting conductivity, Hall, Seebeck, and Nernst coefficients on a single, thin-film sample. Note the temperature gradient for the Seebeck and Nernst measurements and the applied magnetic field for the Hall and Nernst measurements. 
a)
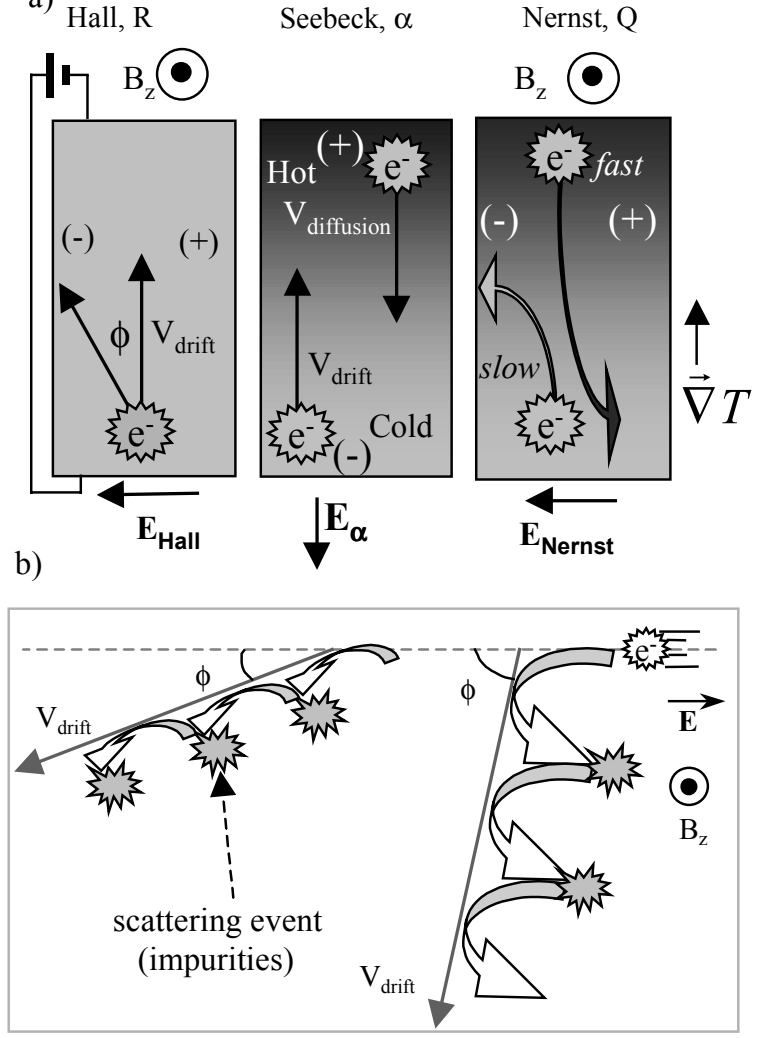

c)

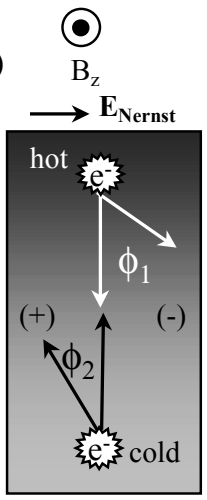

$\phi_{1}>\phi_{2}$

$\tau_{1}>\tau_{2}$

$\mathrm{s}^{\prime}>0$

ionized

impurities

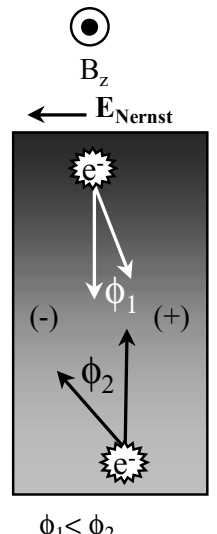

$\phi_{1}<\phi_{2}$

$\tau_{1}<\tau_{2}$

$\mathrm{s}^{\prime}<0$

acoustic

phonons

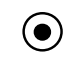

$\mathrm{B}_{z}$

$E_{\text {Nernst }}=0$

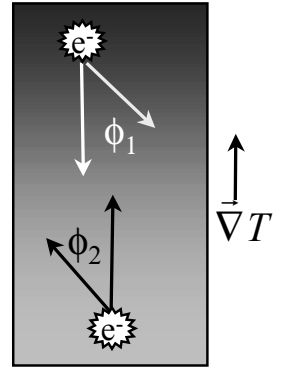

$\phi_{1}=\phi_{2}$

$\tau_{1}=\tau_{2}$

$\mathrm{s}^{\prime}=0$

neutral

impurities

Fig. 3. Part a) Hall, Seebeck, and Nernst transport phenomena for n-type, parabolic band materials exhibiting ionized impurity scattering are shown in cartoon form. The Seebeck effect is an electric field, at steady-state conditions, between the carrier diffusion and drift velocities. The Nernst effect is established by a net negative charge due to the slower moving, "cold" electrons experiencing a greater Lorentz force. Part b) An electron in a crossed electric and magnetic field. The longer relaxation time between collision, scattering events, shown on the right, gives rise to a longer helical arc length and a larger Hall angle, $\phi$. Part c) The Hall angles for the Nernst effect, $\phi_{1}$ and $\phi_{2}$, determine the type of scattering in the material. For ionized impurity scattering, $\phi_{1}$ is larger than $\phi_{2}$. This implies $\tau_{1}$ is greater than $\tau_{2}$ (Part b). With the relaxation-time approximation, equation [3], s' must be positive to give a positive relationship between energy of the carriers (hot) and the relaxation time. For acoustic phonon scattering, $\phi_{2}$ is larger than $\phi_{1}$, leading to $\tau_{1}$ being less than $\tau_{2}$. This implies s' is less than zero for energy and relaxation time to be inversely related. For Neutral impurity scattering, there is no Nernst signal and $\tau$ does not depend on the energy of the carriers, $\mathrm{s}^{\prime}=0$. 


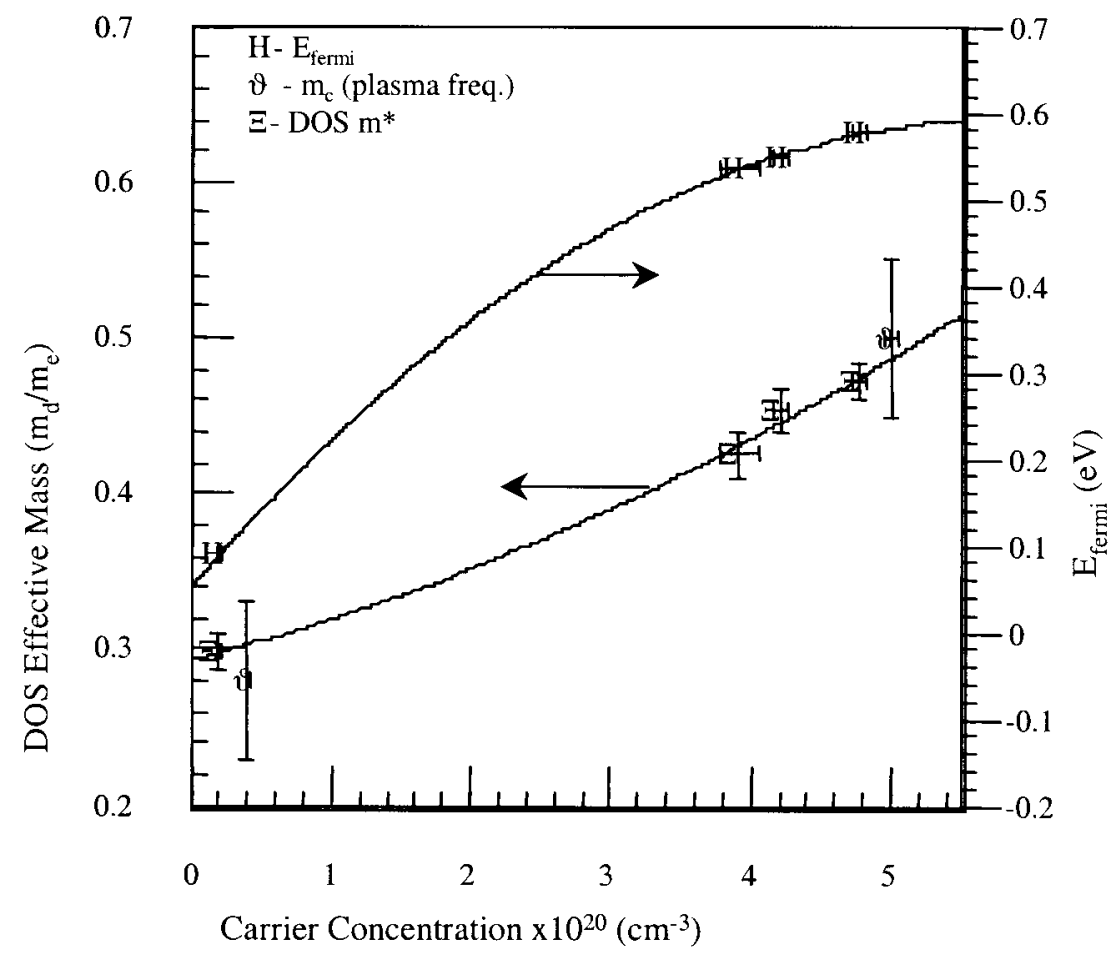

Fig. 4. Density-of-states (DOS) effective mass, Fermi energy level with respect to the conduction band, and plasma frequency - conductivity effective mass, $\mathrm{m}_{\mathrm{c}}$, versus carrier concentration for $\mathrm{ZnO}$ and $\mathrm{ZnO}: \mathrm{Al}$. 

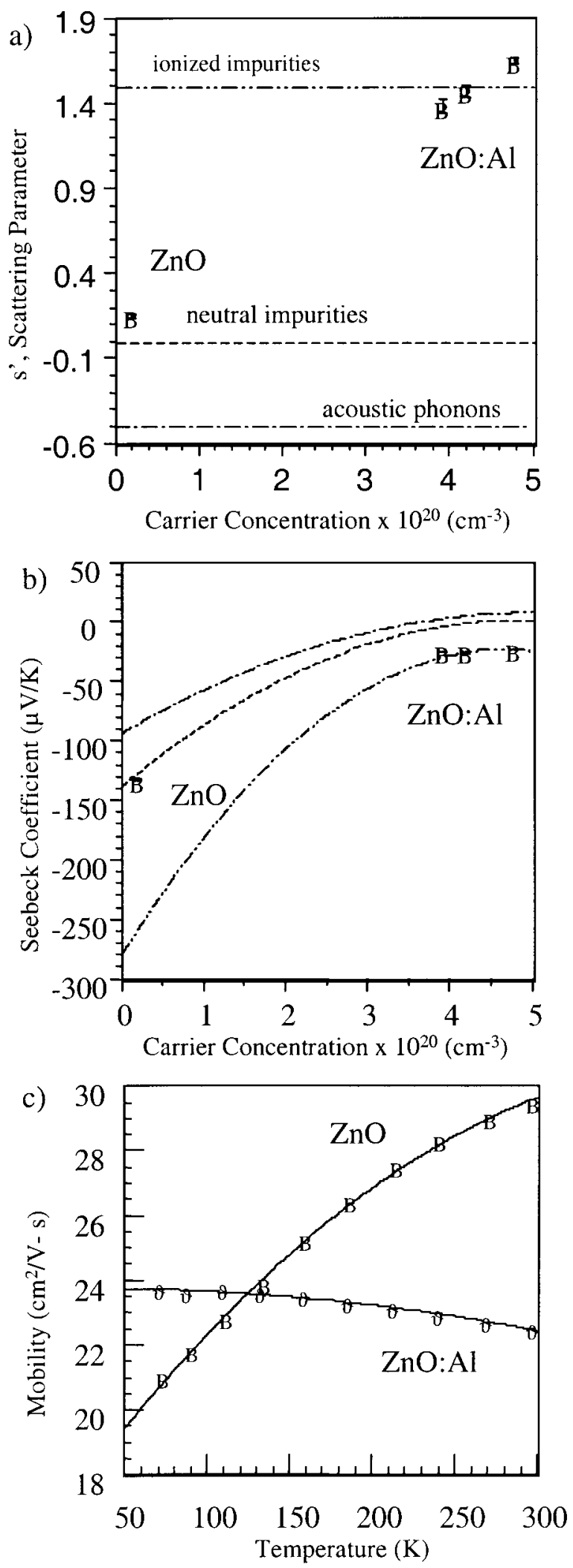

Fig. 5. Two different scattering mechanisms are exhibited in the $\mathrm{ZnO}$ films in this study. The $\mathrm{ZnO}$ :Al films show ionized impurity scattering, whereas the $\mathrm{ZnO}$ film shows neutral impurity scattering. a) Scattering parameter, s vs. n. Data points are plotted, along with theoretical values of s', for three different scattering mechanisms. b) Seebeck coefficient vs. n. Data points are plotted, along with theoretical curves, for three different scattering mechanisms. c) Mobility, $\mu$ vs. $n$. 


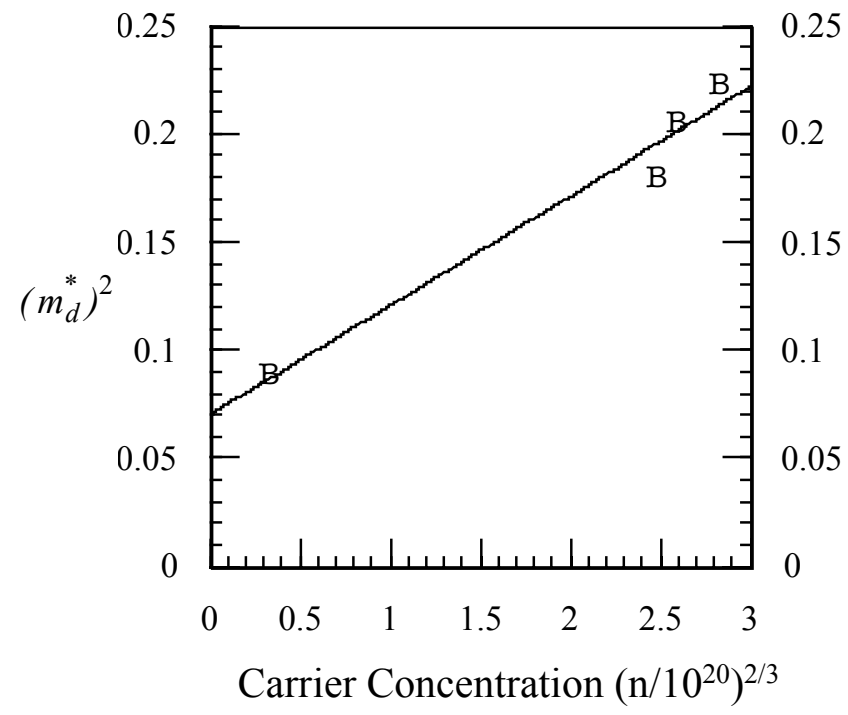

Fig. 6. DOS $\mathrm{m}^{*^{2}}$ vs. $\mathrm{n}^{2 / 3}$. The linear fit of the data justifies our first-order non-parabolicity approximation. The $y-$ intercept gives the square of the effective mass at the bottom of the conduction band. 


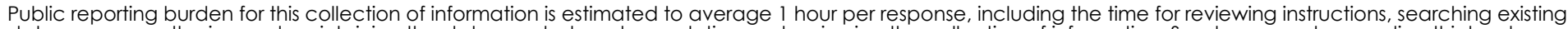

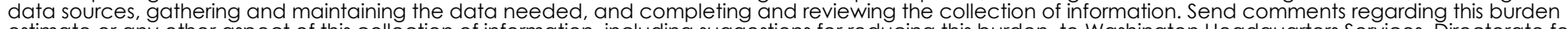

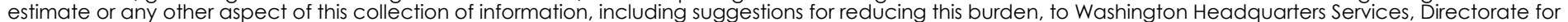

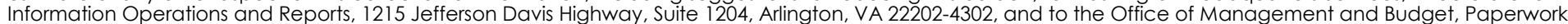
Information Operations and Reports, 1215 Jefferson Davi
Reduction Project (0704-0188), Washington, DC 20503.

\begin{tabular}{|l|l|l|l}
\hline 1. AGENCY USE ONLY (Leave blank) & $\begin{array}{l}\text { 2. REPORT DATE } \\
\text { March } 2000\end{array}$ & $\begin{array}{l}\text { 3. REPORT TYPE AND DATES COVERED } \\
\text { conference paper }\end{array}$ \\
\hline
\end{tabular}

4. TITLE AND SUBTITLE

Direct Measurement of Density-of-States Effective Mass and Scattering Parameter in Transparent

Conducting Oxides Using Second-Order Transport Phenomena

6. AUTHOR(S)

D.L. Young, T.J. Coutts, V.I. Kaydanov, and W.P. Mulligan

7. PERFORMING ORGANIZATION NAME(S) AND ADDRESS(ES)
5. FUNDING NUMBERS

$\mathrm{C}$

TA: WW215201

8. PERFORMING ORGANIZATION REPORT NUMBER

10. SPONSORING/MONITORING AGENCY REPORT NUMBER

CP-520-27747
9. SPONSORING/MONITORING AGENCY NAME(S) AND ADDRESS(ES)

National Renewable Energy Laboratory

1617 Cole Blvd.

Golden, CO 80401-3393

11. SUPPLEMENTARY NOTES

12a. DISTRIBUTION/AVAILABILITY STATEMENT

National Technical Information Service

U.S. Department of Commerce

5285 Port Royal Road

Springfield, VA 22161 12b. DISTRIBUTION CODE

13. ABSTRACT (Maximum 200 words)

The Boltzmann transport equation can be solved to give analytical solutions to the resistivity, Hall, Seebeck, and Nernst coefficients. These solutions may be solved simultaneously to give the density-of-states (DOS) effective mass $\left(m_{d}^{*}\right)$, the Fermi energy relative to either the conduction or valence band, and a scattering parameter that is related to a relaxation time and the Fermi energy. The Nernst coefficient is essential for determining the scattering parameter and, thereby, the effective scattering mechanism(s). We constructed equipment to measure these four transport coefficients simultaneously over a temperature range of 30-350 K for thin, semiconducting films deposited on insulating substrates. We measured these coefficients for rf magnetron-sputtered zinc oxide, both doped and undoped, with aluminum with carrier concentrations in the range of $1 \times 10^{19}-5 \times 10^{20} \mathrm{~cm}^{-3}$. The $\left(m_{d}^{*}\right)$ was not constant over this carrier concentration range: varying from 0.3 to $0.48 \mathrm{~m}_{\mathrm{e}}$, leading us to conclude that zinc oxide has a non-parabolic conduction band. Conductivity effective mass values for zinc oxide matched our $m_{d}^{*}$ values, revealing a single valley, nearly spherical, constant energy surface for zinc oxide. The measured scattering parameter changed from close to zero to 1.5 as the carrier concentrations increased. The scattering parameter, Seebeck, and mobility vs. temperature data support neutral impurity scattering in the undoped material and ionized impurity scattering in the Al-doped $\mathrm{ZnO}$. The transport theory also allows an extrapolation for an $m_{d}^{*}$ value at the bottom of the conduction band, which was found to be $0.27 \mathrm{~m}_{\mathrm{e}}$.

\section{SUBJECT TERMS}

photovoltaics ; transparent conducting oxides ; second-order transport phenomena ; density of states ; effective mass ; scattering parameters 17. SECURITY CLASSIFICATION
OF REPORT Unclassified
18. SECURITY CLASSIFICATION OF THIS PAGE Unclassified

15. NUMBER OF PAGES 16. PRICE CODE

19. SECURITY CLASSIFICATION OF ABSTRACT Unclassified
20. LIMITATION OF ABSTRACT

UL 